\section{Star dust}

David W. Hughes

Dust in the Universe. Edited by M. E. Bailey and D. A. Williams. Cambridge University Press: 1988. Pp.573. £40, $\$ 69.50$.

IN 1784 William Herschel discovered that there were 'holes in the sky', and these black, star-less regions were photographed by E. E. Barnard around the turn of this century. Coupled with the discovery of a general reddening of the light from distant stars, Herschel and Barnard's observations were taken to indicate the presence of small, solid particles pervading the Galaxy. Until a few years ago this dust cloud was regarded as an annoyance that hindered and prevented optical observations of distant regions. Nowadays it is recognized as being an interesting phenomenon in its own right, giving us the chance to study particles of matter that are merely hundreds of microns across and that have existed for aeons in an ultra-high vacuum and at low temperatures. The dust is on the boundary between largemolecule chemistry and small-particle physics.

Recently there have been enormous advances in our ability to detect the infrared radiation from astronomical objects. The successful Infrared Astronomical Satellite (IRAS), new large ground-based infrared telescopes, sensitive CCD detectors and advanced laboratory work have enabled us to put together a comprehensive picture of the dusty regions in the galaxies. So the time was right for a large conference on the subject. The deliberations of just such a conference, held at the University of Manchester in December 1987, are recorded in the book under

\section{New journals review}

This year Nature's annual new journals review supplement will appear in the issue of 28 September. Publishers and learned societies are invited to submit journals for review, taking note that journals which first appeared after June 1987, and which issued at least four separate numbers by the end of April 1989, will be considered.

Those submitting journals for review should promptly send at least four different issues (the first, the most recent and any two others) of each title to: Tim Lincoln, Nature, 4 Little Essex Street, London WC2R 3LF, England.

For further information please see Nature of 4 May (p.24) or telephone Tim Lincoln on 01-836-6633 (011-44-1836-6633 from the United States) extension 2414 review. The 55 papers convey the impression of an extremely lively area of research, and one in which there often seems to be little consensus. Many important and fundamental questions are still in need of an answer.

About one per cent of the mass of our Galaxy is in the form of dust. But is the dust content in equilibrium? Most of it ( 75 per cent) is formed in the expanding atmospheres of cool $\mathrm{M}$ giant stars by the nucleation of refractory atoms, followed by conglomeration. Supernovae, novae, planetary nebulae and $C$ stars add the rest. The size and population is limited by thermal sputtering, sputtering caused by the impacts of energetic ionized molecules and simple grain-grain collisions. Shock waves from supernovae play a large part in these processes. All in all, a few solar masses of dust are being produced and destroyed each year.

The composition of the dust is another problem that occupies many of the contributors. Spectroscopy provides a host of clues. The $2175 \AA$ ultraviolet 'bump' hints at small particles of graphite. The 9.7- $\mu \mathrm{m}$ infrared feature indicates silicates. Features at $3.3,6.2,7.7,8.6$ and $11.3 \mu \mathrm{m}$ are 'unidentified', and researchers variously suggest that they are due to polycyclic aromatic hydrocarbons (that is, dust smelling like coal-tar soap), hydrogenated amorphous carbon and silicate cores with accreted mantles of ice. The spectral features exhibit polarization, indicating that the dust is elongated and aligned. One idea is that particles might contain tiny superparamagnetic grains of magnetite and are thus lined up by galactic magnetic fields.

In the book, much is made of the fact that the dust was certainly present in the nebular cloud that condensed to form our Solar System. So evidence of the dust should be present in meteorites, comets and the zodiacal cloud.

The high quality of the papers in this volume, many of which are invited contributions by world experts, mean that it will be an essential reference work for many astronomers. Dust in the Universe is a very good book which deals with a subject where books - let alone good ones - are few and far between.

David W. Hughes is in the Department of Physics, University of Sheffield, Sheffield S3 $7 R H$, UK

\section{Birth pangs}

Joseph Silk

The Early Universe: Facts and Fiction. By G. Börner. Springer-Verlag: 1988. Pp.439. DM 125, £41, \$69.

IN RECENT years, cosmology has become a respectable field. Five decades ago, many of the writings in this area were characterized by their mix of relativity and philosophy; now the topic is one in which forefront areas in particle physics and observational astronomy are brought together.

The Early Universe is one of the first textbooks to attempt a synthesis of the field from a modern perspective, and Börner is to be congratulated on his brave attempt to introduce the new cosmology to an audience at the level of a first-year graduate student in astrophysics. He tells a gripping story, although the writing is somewhat uneven and the background required of the reader is variable. Feynman diagrams and galaxy rotation curves, Higgs-field lagrangians and Tully-Fisher relations, make odd bedfellows. A kinder, gentler approach to the particle physics theory might have helped overcome the interdisciplinary barriers.

Nonetheless, the book merits close attention. The first four chapters introduce the standard cosmological models and provide a concise, topical review of observational cosmology. We learn about the fundamental facts of cosmology, including determinations of the age of the Universe and of its mean density. Cosmologists have been striving for many years to reconcile facts and theory. Things haven't changed. We still have an age for the oldest globular cluster stars that exceeds, if the Universe is at critical density, the expansion age inferred to be two-thirds of the Hubble time, $H_{0}^{-1}$. To amend the situation, we can introduce a cosmological constant, as thoroughly described by Börner, or if we sympathize with Einstein's view that the cosmological constant was his greatest mistake, look elsewhere for the resolution. One can always criticize the observational age determinations, which present a tempting target, although any hitherto unrecognized sources of systematic bias towards lower globular cluster ages are in short supply.

The main loophole, however, is the premise that the Universe should be at critical density. That premise is based entirely on theoretical and even philosophical prejudice, epitomized by Ernest Barnes, the cosmologically inclined Bishop of Birmingham, who contended that "infinite space is simply a scandal to human thought". All astronomical observations point to an open Universe, destined to expand forever, whose age is $H_{0}^{-1}$, which for the acceptable value $H \approx$ $75 \mathrm{~km} \mathrm{~s}^{-1} \mathrm{Mpc}^{-1}$ one estimates to be about $15 \times 10^{4} \mathrm{yr}$. Such an age is perfectly compatible with all other age determinations.

Another pedestal of the standard model of cosmology, the hot Big Bang, is the cosmic microwave background radiation. The search for possible distortions from a black body spectrum and for deviations 